\title{
A Self-Contained Culture Platform Using Carbon Dioxide Produced from a Chemical Reaction Supports Mouse Blastocyst Development In Vitro
}

\author{
Jason E. SWAIN') \\ ${ }^{1)}$ Department of OB/GYN, Reproductive Sciences Program, University of Michigan, MI 48108, USA
}

\begin{abstract}
Elevated $\mathrm{CO}_{2}$ is required for in vitro embryo culture to maintain proper media pH and to supply embryo metabolic pathways. As an alternative to current approaches using gas cylinders, we examined use of a chemical reaction to supply $\mathrm{CO}_{2}$. A closed culture system was constructed and chemicals added to generate $\mathrm{CO}_{2}$, which was then supplied to developing embryos. This system was shown to provide a stable pH (7.2-7.4) over 4 days of use. One-cell mouse embryos were cultured in the device and no difference in blastocyst formation or cell number was apparent between embryos grown in a closed system with $\mathrm{CO}_{2}$ supplied by a chemical reaction or positive controls grown in a an open system in a $\mathrm{CO}_{2}$ incubator. This approach provides a highly purified, inexpensive, and easily obtainable gas source and offers potential for development of new, self-contained culture platforms.
\end{abstract}

Key words: Blastocyst, Carbon dioxide, $\mathrm{pH}$

(J. Reprod. Dev. 57: 551-555, 2011)

$\mathrm{n}$ attempts to improve efficiency, approaches to in vitro embryo culture have evolved over time. The majority of change has involved manipulations of culture media substrate composition, however, other environmental factors dramatically impact embryo quality, such as culture atmosphere, and also require examination.

While research concerning atmospheric impact on embryo development is not completely lacking, most of the available literature focuses on impact of oxygen concentration [1-7]. Perhaps more importantly, $\mathrm{CO}_{2}$ gas is critical to support embryo development in vitro. Carbon dioxide gas dissolves in the aqueous culture medium to produce carbonic acid, which reaches equilibrium with sodium bicarbonate in the medium to set the external $\mathrm{pH}(\mathrm{pHe})$, which can alter internal $\mathrm{pH}$ (pHi) and subsequent embryo development and function [8-12]. Also, independent of $\mathrm{pHe}, \mathrm{CO}_{2}$ is required for embryo development in vitro, as carbon from external $\mathrm{CO}_{2}$ is fixed by the embryo and used for various metabolic processes [12-14]. Thus, while there are various culture systems which permit embryo development in both open and closed vessels by utilizing zwitterionic buffers to maintain pHe and do not utilize external $\mathrm{CO}_{2}$ supplied by an incubator (see review [15]), when proper comparative studies are performed, embryo development is often compromised in these systems, in part, due to lack of external carbon/ $/ \mathrm{CO}_{2}[16]$.

Though levels of $\mathrm{CO}_{2}$ utilized for embryo culture may vary from laboratory to laboratory, depending upon target $\mathrm{pHe}$ and relevant factors, one consistent variable is that $\mathrm{CO}_{2}$ is supplied via cylinders of compressed gas. This is often true even when utilizing various portable systems using small incubators or closed culture vessels

Received: March 9, 2011

Accepted: April 20, 2011

Published online in J-STAGE: April 30, 2011

(C2011 by the Society for Reproduction and Development

Correspondence: JE Swain (e-mail: swainj@med.umich.edu)
$[17,18]$. This current approach of gas supply can be inefficient and expensive. Liters of gas are pumped into often large incubators to maintain the pHe of microliter volumes of media. Furthermore, maintaining this gas atmosphere in traditional incubators is hindered by repeated openings/closings, which utilizes/wastes more gas. These perturbations in gas environment impact media equilibration and subsequent embryo quality. Additionally, the expense involved in the initial purchase of an incubator and the associated space/supplies to maintain cylinders of gas is problematic, especially if using a portable field system. Furthermore, and perhaps of more concern, purity of gas in cylinders can be questionable and detrimental to embryo development [19].

In an attempt to explore a more efficient alternative to current methods of supplying $\mathrm{CO}_{2}$ for embryo culture, use of a chemical reaction, supplied as Alka Seltzer ${ }^{\circledR}$ tablets, in a closed embryo culture system was examined.

Initial experiments focused on ability to obtain and maintain an appropriate pHe in a closed system with chemically generated $\mathrm{CO}_{2}$. Varying portions of Alka Seltzer ${ }^{\circledR}$ were removed and weighed to allow addition to the close culture system. Traditional bicarbonate media (25 mM NaHCO 3 ) was tested and titrations indicated between 0.025 and $0.05 \mathrm{~g}$ of the tablet are needed to achieve appropriate pHe for embryo culture (7.20-7.40) (Fig. 1A). Additionally, the closed-system apparatus was able to maintain a stable pHe over $96 \mathrm{~h}$, with no significant changes in pHe compared to $24 \mathrm{~h}$ (Fig. 1B) To determine if $\mathrm{pH}$ stability could be improved, similar experiments were performed with dual-buffered HEPES:MOPS media with $25 \mathrm{mM} \mathrm{NaHCO}_{3}$ and similar pHe values were obtained (data not reported). Therefore, $0.03 \mathrm{~g}$ of the tablet was used in subsequent embryo development experiments.

Due to the possibility of limited access to Alka Selzter ${ }^{\circledR}$ and to further define the system, use of the raw chemical active ingredients were also used to generate $\mathrm{CO}_{2}$. The approximate amounts of citric acid (20 mg) and sodium bicarbonate $(20 \mathrm{mg}$ ) found in $0.05 \mathrm{~g}$ 


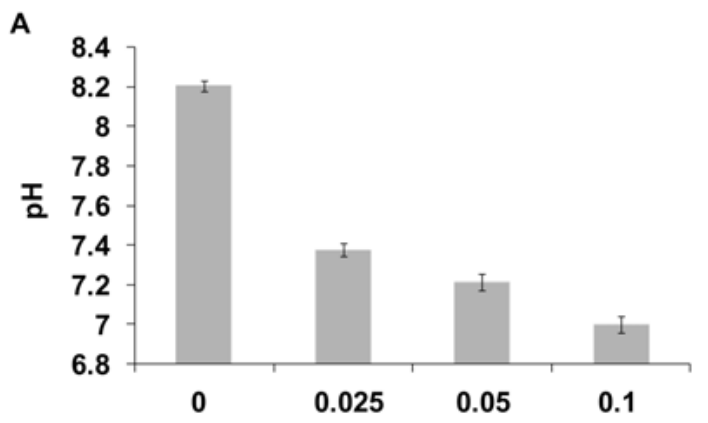

B

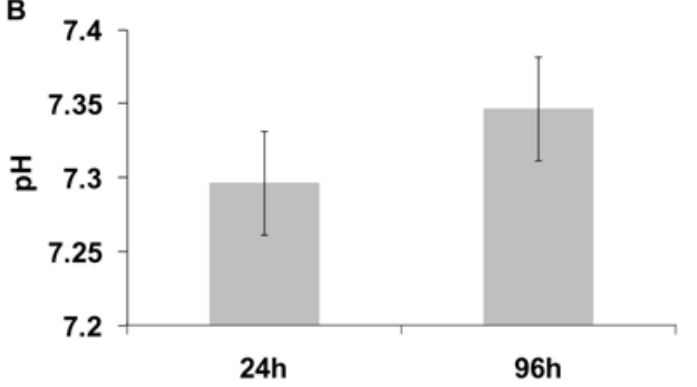

Fig. 1. A: Graphical display of dose titration to determine amount of chemical tablet needed to obtain appropriate media pHe (7.2-7.4) following $24 \mathrm{~h}$ incubation. B: Verification that the closed culture system maintained a stable pHe over $96 \mathrm{~h}$ of incubation using $0.03 \mathrm{~g}$ of tablet. Data are presented as the mean \pm SEM.

of the Alka Seltzer tablet were added to the closed system and resulting pHe measured of bicarbonate buffered media. The pHe of 3 replicates indicate the raw chemicals yielded a pHe of $7.35 \pm 0.02$ (mean \pm SEM).

Examination of embryo development using $0.03 \mathrm{~g}$ of tablet demonstrated no differences in blastocyst formation between embryos grown in positive controls in an open system in a $\mathrm{CO}_{2}$ incubator or treatments, which consisted of KSOMAA or KSOMAA + HEPES:MOPS in a closed system with $\mathrm{CO}_{2}$ supplied by a chemical reaction (92.6, 85.2 vs. 85.2\%, respectively). Negative control embryos grown in a closed system with no $\mathrm{CO}_{2}$ supply yielded significantly lower rates of blastocyst formation compared to treatment or positive control ( $0 \%$ ) (Fig. 2A). Rates of blastocyst hatching were not significantly different between positive controls or treatments (KSOMAA, KSOMAA+HEPES:MOPS), though rates were slightly less in closed test tubes with chemically generated $\mathrm{CO}_{2}$ ( $29.6 \pm 9.3,14.8 \pm 3.9,18.5 \pm 5.3$, respectively) (Fig. 2A). Examination of blastocyst cell numbers between positive controls and treatments (KSOMAA or KSOMAA + HEPES:MOPS) revealed no difference $(47.44 \pm 5.37 n=18,52.81 \pm$ $7.70 n=16,44.7 \pm 5.2 n=14$, respectively) (Fig. 2B).

It is shown here that 1-cell mouse embryos can be cultured to the blastocyst stage using $\mathrm{CO}_{2}$ supplied via a simple chemical reaction. Chemically produced $\mathrm{CO}_{2}$ used in a closed system offers a pure and easily accessible gas source and offers an alternative to traditional incubators and gas cylinders. It is known that purity of ambient atmosphere can impact embryo development and pregnancy [19-

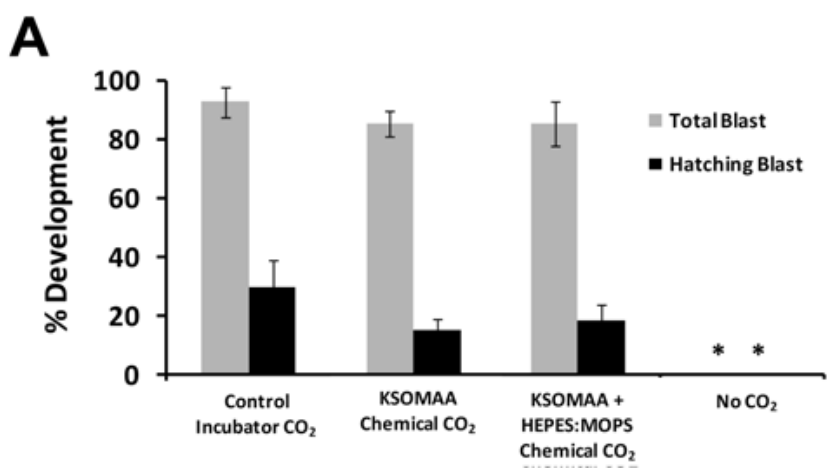

B

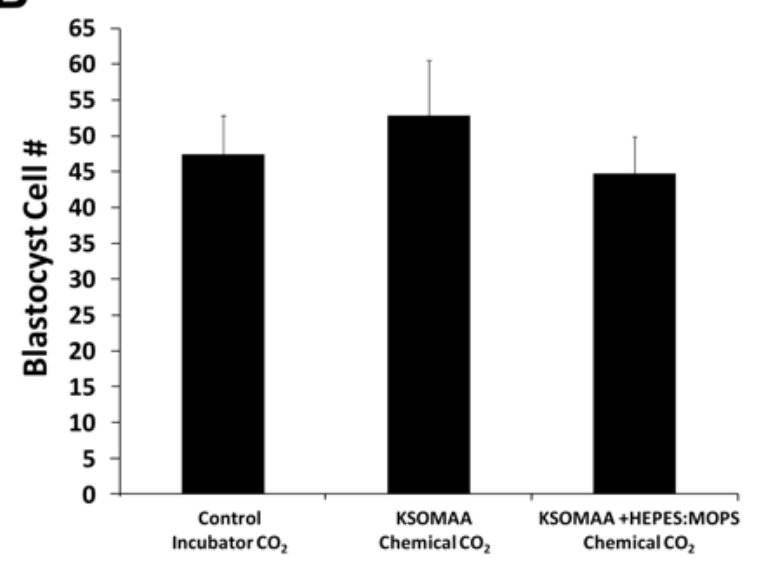

Fig. 2. A: Graphical display of resulting blastocyst development and hatching following culture in $\mathrm{CO}_{2}$ produced via a chemical reaction, cylinders of gas connected to an incubator, or no $\mathrm{CO}_{2}$. B: Total cell number of blastocysts developed from two sources of $\mathrm{CO}_{2}$. Data are presented as the mean \pm SEM. * represents a significant decrease in blastocyst development and hatching compared to other treatments , $\mathrm{P}<0.01$.

21]. The same holds true for supplied gases. Levels of benzene, freons, aldehydes, toluene, acetone and isopropanol have been identified in compressed gas tanks [19]. Though charcoal activated filters in the incubator [22] or in the gas-line can remove some potential contaminants, impurities in atmosphere/gas composition directly from the tank or picked up during transit to the incubator through tubing, can still compromise embryo development. Chemically provided $\mathrm{CO}_{2}$ offers a potential solution to these impurities, as well as an alternate backup supply source of gas if needed due to inability to obtain/store cylinders of gas or equipment failure.

Interestingly, an early study examining ability to culture mouse embryo in vitro utilized exhaled alveolar air as an alternate to supplying external $\mathrm{CO}_{2}$ gas from medical cylinder [23], though this approach limits ability to adjust $\mathrm{CO}_{2}$ levels and pHe. use of $\mathrm{CO}_{2}$ generated from a more complex chemical reaction has been previously used to culture bovine embryos. Prior studies have utilized effervescent granules of tartaric acid, carbonated hydrogen natrium and silicone fiber, granules reacted with water to produce $\mathrm{CO}_{2}$ for use during in vitro oocyte maturation and culture of bovine IVF embryos and nuclear transfer blastocysts [24-27]. In a similar 

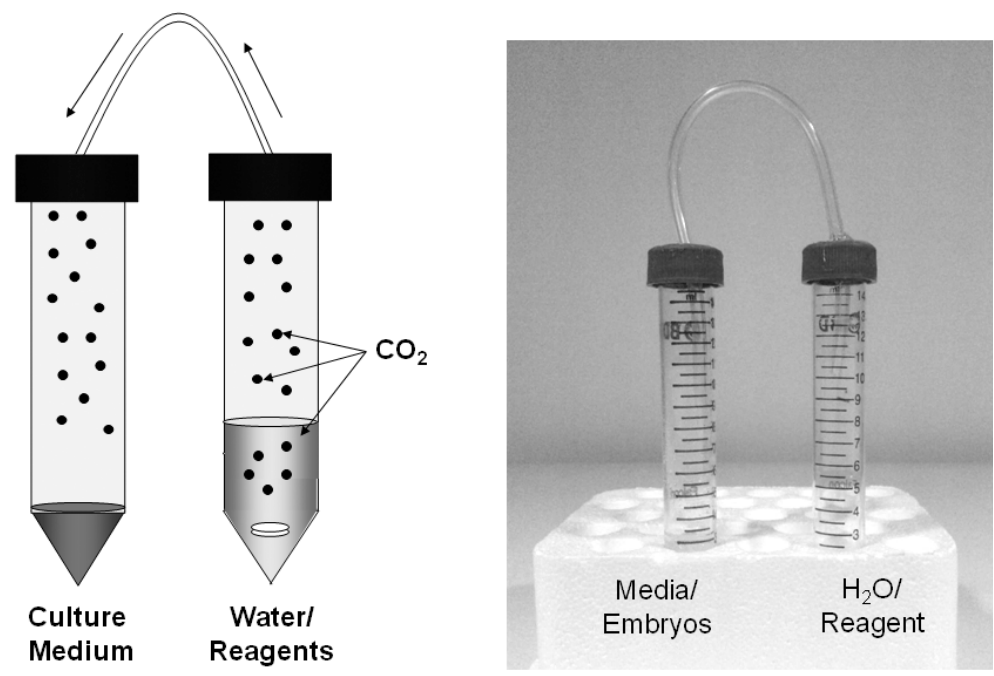

Fig. 3. A: Schematic of the closed culture device and supply of $\mathrm{CO}_{2}$ to media and embryos. B: Image of the culture device.

fashion, bovine embryos were also grown using the $\mathrm{Oxoid}^{\mathrm{TM}}$ atmosphere generating system [28], a commercially available item normally used for growth of microorganisms. This approach utilizes a paper sachet of ascorbic acid and reacts with air to provide an atmosphere of $\sim 6 \% \mathrm{CO}_{2}$ and $15 \%$ oxygen when used in the proper sized Aerojar ${ }^{\mathrm{TM}}$. Importantly, chemicals used in previous studies to generate $\mathrm{CO}_{2}$ are not found in embryo culture media or IVF laboratories, and thus may be difficult to obtain, and are likely embryo toxic. These complex commercial products/reactions may not be widely available or easy to reproduce in a remote laboratory. In contrast, key reagents from our study (citric acid and sodium bicarbonate) are common ingredients in culture media, and thus offer an easily obtainable alternative to prior complex approaches. Products of the chemical reaction (sodium citrate and water), are also common products in culture media and would appear to be a safer alternative than prior chemical approaches. Other acid:base reactions could be utilized in a similar fashion to produce $\mathrm{CO}_{2}$, though potential for toxic fumes or byproducts should be considered. Furthermore, we compared our approach to positive control embryos grown in an incubator and obtained comparable rates of blastocyst development, which, at $>80 \%$ from 1-cell, are considered appropriate when using the widely accepted MEA assay standards of various commercial culture media suppliers. Comparison of blastocyst cell number further support efficacy of the system. Future experiments examining development of embryos from other domestic animal species may be insightful in further examining efficacy of this approach. Additionally, no reports exist regarding transfer of embryos from any species after being grown in conditions using chemically generated $\mathrm{CO}_{2}$, and will likely prove insightful if pursuing further advancement of this approach.

An additional advantage of using the approach of a closed culture platform for $96 \mathrm{~h}$ of uninterrupted culture is maintenance of stable $\mathrm{pH}$ and $\mathrm{CO}_{2}$ levels, providing a more stable culture environment. Opening and closing of large incubators results in evacuation of atmosphere and manipulations of cells in Petri dishes outside the incubator can result in damaging $\mathrm{pH}$ rises. It has been reported that use of closed modular chambers improved mouse embryo development compared to culture in large incubators, presumably due to increased stability of environmental condition [29]. The same may hold true for the closed culture system used in this study. However, one limitation with the rudimentary approach used is the reduced ability to adjust/monitor $\mathrm{CO}_{2}$ levels. We examined used of enhanced buffering media with HEPES:MOPS, but no difference in pHe or development were observed from bicarbonateonly buffered media. Furthermore, monitoring of embryo development is restricted. Though optical clarity of the test tubes does allow for visualization of developing embryos, resolution and detail is limited. Additionally, ability to isolate or monitor individual cells is problematic in the current format. However, adjustments could be made to the platform and incorporation of a closed culture system with chemically generated $\mathrm{CO}_{2}$ in a small sealed Petri dish with individual wells or chambers to house embryos, surrounding a central $\mathrm{CO}_{2}$ generating well would improve visibility and embryo tracking. Devices could be housed in small, simple, warming boxes to maintain temperature, similar to prior approaches at developing portable field incubators, but without the need for external cylinders of gas. This approach may be amenable for development of a self-contained culture platform, perhaps in conjunction with emerging microfluidic technology.

In conclusion, preliminary findings in this technical report demonstrate that chemically generated $\mathrm{CO}_{2}$ from a simple reaction using common laboratory ingredient can be used to efficiently culture mouse embryos and offers the opportunity to supply a pure and inexpensive gas source compared to current traditional techniques. This approach may lend itself to establishment of new field laboratories or offer a back- up gas supply mechanism to current labs. Furthermore, this approach may lead to novel environmentally stable embryo culture platforms, without the use of traditional large 
gas-filled incubators or external gas cylinders.

\section{Methods}

\section{Construction of device}

A closed embryo culture system was constructed consisting of two $15 \mathrm{ml}$ screw-cap test tubes, connected by Tygon tubing fitted through holes in the caps (Fig. 3). Holes were sealed with a silicone adhesive and allowed to off-gas.

\section{Chemical titration and $\mathrm{pH}$ measurement}

To initially determine approximate amount of chemical reagent needed to obtain appropriate $\mathrm{pH}(7.20-7.40), 1 \mathrm{ml}$ of culture medium (KSOMAA; Ho et al. 1995) + 10\% Serum Substitute Supplement (SSS, Irvine Scientific, Irvine, CA, USA) was place into one tube, while $3 \mathrm{ml}$ of $\mathrm{H}_{2} \mathrm{O}$ was placed into the second tube. Varying amounts of reagent $(0.025,0.05,0.1 \mathrm{~g})$ were obtained by breaking small portions from the large Alka Seltzer ${ }^{\circledR}$ tablet, then added to the tube containing $\mathrm{H}_{2} \mathrm{O}$ to generate $\mathrm{CO}_{2}$, which was subsequently supplied to the tube containing culture medium by the connecting tubing. The chemical reaction utilizes the acid:base reaction : $\mathrm{HCO}_{3}(\mathrm{aq})+\mathrm{H}^{+}(\mathrm{aq}) \rightarrow \mathrm{H}_{2} \mathrm{O}(\mathrm{l})+\mathrm{CO}_{2}$ (g) and goes to completion in seconds Therefore, for ease of reagent measurement and ability to add reagents quickly to the tube in a repeatable fashion before closing, the majority of subsequent experiments utilized small portions obtained from the larger tablet of Alka Seltzer ${ }^{\circledR}$. Each whole tablet contains $1000 \mathrm{mg}$ citric acid , $325 \mathrm{mg}$ acetylsalicylic and $1918 \mathrm{mg}$ of bicarbonate. Because each tablet weighs $\sim 3.24 \mathrm{~g}$, the amounts of tablet used $(0.025,0.05$ and $0.1 \mathrm{~g})$ contain 10.2, 20.4 and $40.9 \mathrm{mg}$ of total acid, respectively, which reacts with equal amounts of sodium bicarbonate (1:1 ratio). Excess bicarbonate in the tablet is not utilized in generating $\mathrm{CO}_{2}$. Tubes were placed into a $37 \mathrm{C}$ ungased warming oven and $\mathrm{pHe}$ of culture media measured $\sim 24 \mathrm{~h}$ and later to allow sufficient equilibration.

Furthermore, to further define the chemical reaction for greater reproducibility, use of $20 \mathrm{mg}$ of citric acid and sodium bicarbonate, were enclosed in a cellulose capsule and added to the closed system to verify whether these raw reagents could reproduce the reaction and provide a reproducible $\mathrm{pHe}$ in the range of 7.2-7.4, similar to use of $0.05 \mathrm{~g}$ of the tablet. Encapsulation of the reagents allowed for easy addition to the $\mathrm{H}_{2} \mathrm{O}$ tube, permitting the tube to be closed before the chemical reaction could proceed/complete, thereby improving repeatability.

Based on initial dose titrations using small pieces of tablet, subsequently $0.03 \mathrm{~g}$ of Alka Selzter was utilized for the remainder of the developmental experiments. pHe was measured following $24 \mathrm{~h}$ of culture and to verify pHe stability in the closed-system device, $\mathrm{pHe}$ was measured in a second tube $96 \mathrm{~h}$ later to verify it remained within the 7.2-7.4 desired range. pHe of all media was measured directly within the test tubes following electrode calibration with $\mathrm{pH}$ standards (7 and 10) using a double junction glass $\mathrm{KCl}$ filled electrode warmed to $37 \mathrm{C}$. Experiments were performed 3-5 times and $\mathrm{pHe}$ values averaged. Data are presented as the mean $\pm \mathrm{SEM}$.

\section{Embryo culture}

Subsequently, $0.03 \mathrm{~g}$ of the Alka Seltzer reagent was used to equilibrate $1 \mathrm{ml}$ of media for $24 \mathrm{~h}$ prior to adding frozen/thawed 1cell mouse embryos (B6C3F1; Embryotech Havervill, MA, USA).
Five to six embryos were cultured per tube in pre-equilibrated medium, an additional $0.03 \mathrm{~g}$ of reagent was then added to the $\mathrm{H}_{2} \mathrm{O}$ and tubes re-capped. To reduce variability and ensure appropriate atmosphere, the tube containing the embryos was capped first, while the tube containing the water and chemicals was capped second, immediately following addition of the reagent tablet. Treatments included KSOMAA $+10 \%$ SSS and a double-buffered medium consisting of KSOMAA +10\%SSS+10 mM HEPES + 10 $\mathrm{mM}$ MOPS buffers to help further stabilize medium pHe. Both media contained $25 \mathrm{mM}$ bicarbonate and pHe values were similar. Following $96 \mathrm{~h}$ of uninterrupted culture, in order to maintain the $\mathrm{CO}_{2}$ atmosphere and $\mathrm{pHe}$, embryos were removed, graded for development, and assessed for total cell number. Positive controls consisted of an unsealed test tube of KSOMAA $+10 \%$ SSS in a $\mathrm{CO}_{2}$ incubator ( $\mathrm{pH}$ 7.2-7.4), while negative controls consisted of a sealed test tube and no $\mathrm{CO}_{2}(\mathrm{pH}$ 8.1-8.3). Each treatment utilized a total of 27 embryos, data were collected over 5 replicates and presented as the mean \pm SEM.

\section{Blastocyst cell count}

For total cells counts, blastocysts were exposed to $0.9 \%$ sodium citrate for $\sim 5$ min and then placed into methanol:acetic acid:water (3:2:1) for $\sim 10$ sec. Blastocsyts were placed onto a microscope slide and allowed to air-dry before placement in methanol:acetic acid (3:1) for $24 \mathrm{~h}$. Slides were stained for $10 \mathrm{~min}$ with acetoorcein and cells counted on a brightfield microscope. Data were analyzed using ANOVA followed by Bonferroni multiple comparison test.

\section{References}

1. Bavister B. Oxygen concentration and preimplantation development. Reprod Biomed Online 2004; 9: 484-486.

2. Harvey AJ. The role of oxygen in ruminant preimplantation embryo development and metabolism. Anim Reprod Sci 2007; 98: 113-128.

3. Meintjes M, Chantilis SJ, Douglas JD, Rodriguez AJ, Guerami AR, Bookout DM, Barnett BD, Madden JD. A controlled randomized trial evaluating the effect of lowered incubator oxygen tension on live births in a predominantly blastocyst transfer program. Hum Reprod 2009; 24: 300-307.

4. Waldenstrom U, Engstrom AB, Hellberg D, Nilsson S. Low-oxygen compared with high-oxygen atmosphere in blastocyst culture, a prospective randomized study. Fertil Steril 2009; 91: 2461-2465.

5. Kovacic B, Sajko MC, Vlaisavljevic V. A prospective, randomized trial on the effect of atmospheric versus reduced oxygen concentration on the outcome of intracytoplasmic sperm injection cycles. Fertil Steril 2010; 94: 511-519

6. Kovacic B, Vlaisavljevic $\mathbf{V}$. Influence of atmospheric versus reduced oxygen concentration on development of human blastocysts in vitro: a prospective study on sibling oocytes. Reprod Biomed Online 2008; 17: 229-236.

7. Ciray HN, Aksoy T, Yaramanci K, Karayaka I, Bahceci M. In vitro culture under physiologic oxygen concentration improves blastocyst yield and quality: a prospective randomized survey on sibling oocytes. Fertil Steril 2009; 91(4 Suppl): 1459-1461.

8. Kane MT. Bicarbonate requirements for culture of one-cell rabbit ova to blastocysts. Biol Reprod 1975; 12: 552-555.

9. Squirrell JM, Lane M, Bavister BD. Altering intracellular $\mathrm{pH}$ disrupts development and cellular organization in preimplantation hamster embryos. Biol Reprod 2001; 64: $1845-1854$.

10. Lane M, Lyons EA, Bavister BD. Cryopreservation reduces the ability of hamster 2cell embryos to regulate intracellular pH. Hum Reprod 2000; 15: 389-394.

11. Edwards LJ, Williams DA, Gardner DK. Intracellular $\mathrm{pH}$ of the preimplantation mouse embryo: effects of extracellular $\mathrm{pH}$ and weak acids. Mol Reprod Dev 1998; 50 434-442.

12. Quinn P, Wales RG. Fixation of carbon dioxide by preimplantation rabbit embryos in vitro. J Reprod Fertil 1974; 36: 29-39. 
13. Pike IL, Murdoch RN, Wales RG. The incorporation of carbon dioxide into the major classes of RNA during culture of the preimplantation mouse embryo. J Reprod Fertil 1975; 45: 211-226

14. Graves CN, Biggers JD. Carbon dioxide fixation by mouse embryos prior to implantation. Science 1970; 167: 1506-1508.

15. Swain JE. Optimizing the culture environment in the IVF laboratory: impact of $\mathrm{pH}$ and buffer capacity on gamete and embryo quality. Reprod Biomed Online 2010; 21: 616.

16. Swain JE, Pool TB. New pH-buffering system for media utilized during gamete and embryo manipulations for assisted reproduction. Reprod Biomed Online 2009; 18: 799810.

17. Bavister BD, Poole KA. Duration and temperature of culture medium equilibration affect frequency of blastocyst development. Reprod Biomed Online 2005; 10: 124-129.

18. Vajta G, Bartels P, Joubert J, de la Rey M, Treadwell R, Callesen H. Production of a healthy calf by somatic cell nuclear transfer without micromanipulators and carbon dioxide incubators using the Handmade Cloning (HMC) and the Submarine Incubation System (SIS). Theriogenology 2004; 62: 1465-1472.

19. Hall J, Gilligan A, Schimmel T, Cecchi M, Cohen J. The origin, effects and control of air pollution in laboratories used for human embryo culture. Hum Reprod 1998; 13 (Suppl 4): 146-155.

20. Cohen J, Gilligan A, Esposito W, Schimmel T, Dale B. Ambient air and its potential effects on conception in vitro. Hum Reprod 1997; 12: 1742-1749.

21. Cohen J, Gilligan A, Willadsen S. Culture and quality control of embryos. Hum Reprod 1998; 13 (Suppl 3): 137-144; discussion 145-137.
22. Merton JS, Vermeulen ZL, Otter T, Mullaart E, de Ruigh L, Hasler JF. Carbon-activated gas filtration during in vitro culture increased pregnancy rate following transfer of in vitro-produced bovine embryos. Theriogenology 2007; 67: 1233-1238.

23. Tarkowski AK, Wroblewska J. Development of blastomeres of mouse eggs isolated at the 4- and 8-cell stage. J Embryol Exp Morphol 1967; 18: 155-180.

24. Suzuki T, Sumantri C, Khan NH, Murakami M, Saha S. Development of a simple, portable carbon dioxide incubator for in vitro production of bovine embryos. Anim Reprod Sci 1999; 54: 149-157.

25. Varisanga MD, Dong YJ, Mtango NR, Fahrudin M, Ni Wayan KK, Suzuki T. Bovine blastocysts obtained from reconstructed cytoplast and karyoplasts using a simple portable $\mathrm{CO}_{2}$ incubator. Cloning 2000; 2: 167-173.

26. Varisanga MD, Dong YJ, Mtang NR, Suzuki T. Comparison of the effects of using standard and simple portable $\mathrm{CO}_{2}$ incubators on the in vitro developmental competence of bovine embryos reconstituted by somatic cell nuclear transfer. Theriogenology 2002; 58: 77-86

27. Dong YJ, Varisanga MD, Mtango NR, Aono M, Otoi T, Suzuki T. Improvement of the culture conditions for in vitro production of cattle embryos in a portable $\mathrm{CO}_{2}$ incubator. Reprod Domest Anim 2001; 36: 313-318.

28. Avery B, Melsted JK, Greve T. A novel approach for in vitro production of bovine embryos: use of the oxoid atmosphere generating system. Theriogenology 2000; 54 1259-1268

29. Gardner DK, Lane M. Alleviation of the '2-cell block' and development to the blastocyst of CF1 mouse embryos: role of amino acids, EDTA and physical parameters. Hum Reprod 1996; 11: 2703-2712. 